\title{
THE ACTION OF HECKE OPERATORS ON HYPERGEOMETRIC FUNCTIONS
}

\section{VICTOR H. MOLL ${ }^{凶}$, SINAI ROBINS and KIRK SOODHALTER}

\author{
(Received 26 September 2009; accepted 9 July 2010)
}

Communicated by I. E. Shparlinski

\begin{abstract}
We study the action of the Hecke operators $U_{n}$ on the set of hypergeometric functions, as well as on formal power series. We show that the spectrum of these operators on the set of hypergeometric functions is the set $\left\{n^{a}: n \in \mathbb{N}, a \in \mathbb{Z}\right\}$, and that the polylogarithms play an important role in the study of the eigenfunctions of the Hecke operators $U_{n}$ on the set of hypergeometric functions. As a corollary of our results on simultaneous eigenfunctions, we also obtain an apparently unrelated result regarding the behavior of completely multiplicative hypergeometric coefficients.
\end{abstract}

2000 Mathematics subject classification: primary 11F25; secondary 33C05.

Keywords and phrases: Hecke operators, hypergeometric functions, eigenvalues, completely multiplicative.

\section{Introduction}

For each $n \in \mathbb{N}$, the space of formal power series $\mathfrak{F}$,

$$
\mathfrak{F}:=\left\{f(x)=\sum_{k=0}^{\infty} c_{k} x^{k}: c_{k} \in \mathbb{C}\right\},
$$

admits the action of the linear operators $U_{n}$ and $V_{n}$ :

$$
\left(U_{n} f\right)(x):=\sum_{k=0}^{\infty} c_{n k} x^{k}
$$

and

$$
\left(V_{n} f\right)(x):=f\left(x^{n}\right)=\sum_{k=0}^{\infty} c_{k} x^{n k} .
$$

The spectral properties of these operators become more interesting when one considers their action on spaces with additional structure.

The work of the first author was partially funded by NSF-DMS 0713836. The work of the second author was partially funded by the Singapore SUG grant given by Nanyang Technological University.

(C) 2010 Australian Mathematical Publishing Association Inc. 1446-7887/2010 \$16.00 
Historically, Hecke studied the vector spaces of modular forms of a fixed weight (see [2]), in which the set $\mathfrak{F}$ is replaced by the space $\mathfrak{M}_{k}$ of analytic functions in the upper half-plane $\mathbb{H}:=\{\tau: \operatorname{Im} \tau>0\}$ that satisfy the condition

$$
f\left(\frac{a \tau+b}{c \tau+d}\right)=(c \tau+d)^{k} f(\tau),
$$

for every matrix in the modular group

$$
\Gamma:=\left\{\left(\begin{array}{ll}
a & b \\
c & d
\end{array}\right): a, b, c, d \in \mathbb{Z}, a d-b c=1\right\} .
$$

These forms are also required to have an expansion at $\tau=i \infty$, or equivalently a Taylor series about $q=0$ :

$$
f(\tau)=\sum_{n=0}^{\infty} c(n) q^{n}
$$

expressed in terms of the parameter $q=\exp (2 \pi i \tau)$.

Hecke introduced a family of operators $T_{n}$, where $n \in \mathbb{N}$, which map the space $\mathfrak{M}_{k}$ into itself. The standard definition is

$$
\left(T_{n} f\right)(\tau):=n^{k-1} \sum_{d \mid n} d^{-k} \sum_{b=0}^{d-1} f\left(\frac{n \tau+b d}{d^{2}}\right),
$$

which, in the special case where $n$ is a prime $p$, becomes

$$
\left(T_{p} f\right)(\tau)=p^{k-1} f(p \tau)+\frac{1}{p} \sum_{b=0}^{p-1} f\left(\frac{\tau+b}{p}\right) .
$$

In terms of the Fourier expansion of $f \in \mathfrak{M}_{k}$, given by

$$
f(\tau)=\sum_{m=0}^{\infty} c(m) q^{m}
$$

the action of $T_{n}$ is

$$
\left(T_{n} f\right)(\tau)=\sum_{m=0}^{\infty} \gamma_{n}(m) q^{m}
$$

where

$$
\gamma_{n}(m)=\sum_{d \mid(n, m)} d^{k-1} c\left(\frac{m n}{d^{2}}\right) .
$$

In particular, when $n$ is a prime $p$,

$$
\gamma_{p}(m)= \begin{cases}c(\mathrm{pm})+p^{k-1} c(\mathrm{~m} / \mathrm{p}) & \text { if } p \text { divides } m \\ c(\mathrm{pm}) & \text { if } p \text { does not divide } m .\end{cases}
$$

History has shown that the study of Hecke operators is one of the most important tools in modern number theory, yielding results about the uniform distributions of 
points, the eigenvalues of Laplacians on various domains, the asymptotic analysis of Fourier coefficients of modular forms, and other branches of number theory.

Interesting results were obtained in the last decade when the space of modular forms was replaced with the space of rational functions (see [4-6]). For example, the spectral properties of the operator $U_{n}$ acting on rational functions were completely characterized, and corollaries about completely multiplicative functions that satisfy linear recurrence sequences were obtained (see [5]).

For the space $\mathfrak{R}$ of rational functions, the coefficients $a_{n}$ in (1.1) are the Taylor coefficients of $A / B \in \mathfrak{R}$, where $B(x)=1+\alpha_{1} x+\cdots+\alpha_{d} x^{d}$, and $A$ is a polynomial of degree less than $d$. The coefficients $a_{n}$ are known to satisfy the recurrence relation

$$
a_{n+d}=-\alpha_{1} a_{n+d-1}-\cdots-\alpha_{d} a_{n}
$$

(see [7] for details). Thus the study of these coefficients employs the theory of linear recurrence sequences and their explicit solutions. One of the main results in [5] is the complete determination of the spectrum of $U_{n}$ acting on $\mathfrak{R}$, namely

$$
\operatorname{spec}\left(U_{n}\right)=\left\{ \pm n^{k}: k \in \mathbb{N}\right\} \cup\{0\} .
$$

Recent work has produced a description of the corresponding rational eigenfunctions $($ see $[3,6])$.

In this paper, we consider the action of $U_{n}$ on the set $\mathfrak{H}$ of hypergeometric functions:

$$
\mathfrak{H}:=\left\{\sum_{k=0}^{\infty} c_{k} x^{k}: \frac{c_{k+1}}{c_{k}} \text { is a rational function of } k\right\} .
$$

We emphasize here that $\mathfrak{H}$ is a set rather than a vector space, because in general the sum of two hypergeometric functions is not another hypergeometric function. Nevertheless, this set includes many of the classical functions as well as all functions of the form

$$
\sum_{k=0}^{\infty} R(k) x^{k}
$$

where $R$ is a rational function.

Every hypergeometric function that we consider has a canonical Taylor series representation of the form

$$
{ }_{p} F_{q}(\mathbf{a}, \mathbf{b} ; x):=\sum_{k=0}^{\infty} \frac{\left(a_{1}\right)_{k}\left(a_{2}\right)_{k} \cdots\left(a_{p}\right)_{k}}{\left(b_{1}\right)_{k}\left(b_{2}\right)_{k} \cdots\left(b_{q}\right)_{k}} \frac{x^{k}}{k !},
$$

where $\mathbf{a}:=\left(a_{1}, a_{2}, \ldots, a_{p}\right) \in \mathbb{C}^{p}$ and $\mathbf{b}:=\left(b_{1}, b_{2}, \ldots, b_{q}\right) \in \mathbb{C}^{q}$ are the parameters of ${ }_{p} F_{q}$. These parameters satisfy $-b_{i} \notin \mathbb{N}$. Here we use the standard notation for the ascending factorial

$$
(c)_{k}:=c(c+1)(c+2) \cdots(c+k-1),
$$

and $(c)_{0}:=1$. For example, $(1)_{k}=k !$, and $(0)_{k}=0$. 
Hypergeometric functions include

$$
f(x)=\sum_{k=0}^{\infty} \frac{x^{k}}{k^{2}+1},
$$

as well as most of the elementary functions. For example, the hypergeometric representation of the exponential function is given by $e^{x}={ }_{1} F_{1}(a, a ; x)$ for any nonzero $a \in \mathbb{C}$. Similarly, the error function

$$
\operatorname{erf}(x)=\frac{2}{\sqrt{\pi}} \int_{0}^{x} e^{-t^{2}} d t
$$

can also be represented as a hypergeometric function, namely

$$
\operatorname{erf}(x)=\frac{2 x}{\sqrt{\pi}}{ }_{1} F_{1}\left(\frac{1}{2}, \frac{3}{2} ; x\right) \text {. }
$$

For a more complete discussion of hypergeometric functions, see [1].

In Section 5 we describe the action of the Hecke operator $U_{n}$ on hypergeometric functions. To state the results, define

$$
\mathfrak{F}_{(p, q)}^{j}:=\left\{x_{p}^{j} F_{q}(\mathbf{a}, \mathbf{b} ; x): \mathbf{a} \in \mathbb{C}^{p}, \mathbf{b} \in \mathbb{C}^{q}\right\},
$$

for fixed $j \in \mathbb{N}$ and $p, q \in \mathbb{N}$. This is the set of all hypergeometric functions that vanish to order $j$ at the origin, and have hypergeometric coefficients

$$
\frac{\left(a_{1}\right)_{k}\left(a_{2}\right)_{k} \cdots\left(a_{p}\right)_{k}}{\left(b_{1}\right)_{k}\left(b_{2}\right)_{k} \cdots\left(b_{q}\right)_{k}}
$$

with $p$ ascending factorials in the numerator and $q$ ascending factorials in the denominator. Observe that

$$
\mathfrak{H}:=\left\{x_{p}^{j} F_{q}(\mathbf{a}, \mathbf{b} ; x): j, p, q \in \mathbb{N}, \mathbf{a} \in \mathbb{C}^{p}, \mathbf{b} \in \mathbb{C}^{q}\right\}=\bigcup_{j, p, q \in \mathbb{N}} \mathfrak{F}_{(p, q)}^{j} .
$$

We establish first the identities

$$
U_{n}\left(x_{p}^{j} F_{q}(\mathbf{a}, \mathbf{b} ; x)\right)=x^{j / n} \sum_{k=0}^{\infty} n^{n k(p-q-1)} \frac{\left(c_{1}\right)_{k}\left(c_{2}\right)_{k} \cdots\left(c_{n p}\right)_{k}}{\left(d_{1}\right)_{k}\left(d_{2}\right)_{k} \cdots\left(d_{n(q+1)-1}\right)_{k}} \frac{x^{k}}{k !}
$$

in $\mathfrak{F}_{\left(p_{1}, q_{1}\right)}^{j}$, when $n$ divides $j$, and

$$
U_{n}\left(x_{p}^{j} F_{q}(\mathbf{a}, \mathbf{b} ; x)\right)=x^{1+\lfloor j / n\rfloor} \sum_{k=0}^{\infty} n^{n k(p-q-1)} \frac{\left(c_{1}\right)_{k} \cdots\left(c_{p n}\right)_{k}}{\left(d_{1}\right)_{k} \cdots\left(d_{(q+1) n-1}\right)_{k}} \frac{x^{k}}{k !}
$$

in $\mathfrak{F}_{\left(p_{1}, q_{1}\right)}^{j}$, if $n$ does not divide $j$. Here $p_{1}=n p$ and $q_{1}=n(q+1)-1$, while the new parameters $\mathbf{c}$ and $\mathbf{d}$ are given in (5.1) and (5.2). In particular, we observe in Section 5 that $U_{n}$ maps $\mathfrak{F}_{(p, q)}^{j}$ into itself if and only if $p=q+1$. These are the balanced hypergeometric functions. Therefore, an eigenfunction of $U_{n}$ must satisfy the condition $p=q+1$. 
The eigenfunctions of $U_{n}$ on the space of formal power series are described in Section 4. We consider solutions of $U_{n} f=\lambda f$, where $f(x)=x^{j} \sum_{k=0}^{\infty} a_{k} x^{k}$, and show that if $n$ divides $j$, then it follows that $j=0, \lambda=1$ and the eigenfunction $f$ must reduce to the rational function $1 /(1-x)$. On the other hand, in the case where $n$ does not divide $j$, we show that $j$ must be 1 and the eigenvalue $\lambda$ must be of the form $n^{a}$, where $a \in \mathbb{Z}$.

One of the main results here is the complete characterization of the spectrum $\operatorname{spec}\left(U_{n}\right)$ of $U_{n}$ on hypergeometric functions, yielding the result that

$$
\operatorname{spec}\left(U_{n}\right)=\left\{n^{k}: k \in \mathbb{Z}\right\} \cup\{0\} .
$$

As a corollary, we obtain a number-theoretic characterization of all completely multiplicative functions that are also hypergeometric ratios of ascending factorials.

An ultimate goal is to determine the spectrum of all linear combinations of hypergeometric series, but this seems far from the reach of current technology. Thus we focus first on the action of the Hecke operators on a single hypergeometric series.

\section{A natural inner product on $\mathfrak{H}$}

The set $\mathfrak{H}$ of all hypergeometric functions can be endowed with a natural inner product:

$$
\langle f, g\rangle_{R}:=\oint_{|z|=R} f(w) \overline{g(w)} \frac{d w}{w} .
$$

We fix a real number $R$ such that $0<R<1$, so that this inner product is now a function of $R$; as we shall see shortly, it is in fact a real analytic function of $R$. Moreover, we shall also see below that $V_{n}$ is the natural conjugate linear operator to $U_{n}$ with respect to this inner product. This fact is our motivation for introducing the linear operator $V_{n}$.

The next result describes this inner product in terms of the Taylor series expansions of $f$ and $g$.

LEMMA 2.1. If $f(z)=\sum_{n=0}^{\infty} c_{n} z^{n}$ and $g(z)=\sum_{n=0}^{\infty} d_{n} z^{n}$, then

$$
\langle f, g\rangle_{R}=2 \pi i \sum_{n=0}^{\infty} c_{n} \overline{d_{n}} R^{2 n}
$$

PROOF. By definition,

$$
\begin{aligned}
\langle f, g\rangle_{R} & =\oint_{|w|=R} \sum_{n=0}^{\infty} c_{n} w^{n} \times \sum_{m=0}^{\infty} \bar{d}_{m} \bar{w}^{m} \frac{d w}{w} \\
& =\oint_{|w|=R} \sum_{n, m=0}^{\infty} c_{n} \bar{d}_{m} w^{n-m-1} R^{2 m} d w
\end{aligned}
$$

because $\bar{w}=R^{2} / w$ on the circle of integration. Cauchy's integral formula shows that $n=m$, for otherwise the ensuing line integral is zero. This condition gives us the desired result. 
This inner product makes sense even for formal power series, although we restrict our attention to the set $\mathfrak{H}$ of hypergeometric functions. On $\mathfrak{H}$, the inner product of any two hypergeometric functions is in fact a hypergeometric function of $R$, as is easily seen by noting that the product of two hypergeometric coefficients is another hypergeometric coefficient.

To further develop the algebra of the operators $U_{n}$ and $V_{n}$, we now show that $V_{n}$ is the natural conjugate linear operator for $U_{n}$, relative to the inner product introduced above.

LEMMA 2.2. Let $f, g \in \mathfrak{H}$. Then

$$
\left\langle U_{n} f, g\right\rangle_{R}=\left\langle f, V_{n} g\right\rangle_{R^{n}} .
$$

PROOF. On the one hand,

$$
\begin{aligned}
\left\langle U_{n} f, g\right\rangle_{R} & =\left\langle\sum_{k=0}^{\infty} c_{k n} z^{k}, \sum_{k=0}^{\infty} d_{k} z^{k}\right\rangle_{R} \\
& =\sum_{k=0}^{\infty} c_{n k} \overline{d_{k}} R^{2 k} .
\end{aligned}
$$

On the other hand,

$$
\begin{aligned}
\left\langle f, V_{n} g\right\rangle_{R} & =\left\langle\sum_{k=0}^{\infty} c_{k} z^{k}, \sum_{k=0}^{\infty} d_{k} z^{k n}\right\rangle_{R} \\
& =\sum_{k=0}^{\infty} c_{k} \overline{h_{k}} R^{2 k},
\end{aligned}
$$

where we define

$$
h_{k}= \begin{cases}0 & \text { if } n \text { does not divide } k \\ d_{k / n} & \text { if } n \text { divides } k\end{cases}
$$

It follows that

$$
\left\langle f, V_{n} g\right\rangle_{R}=\sum_{k=0}^{\infty} c_{k n} \overline{d_{k}}\left(R^{n}\right)^{2 k}=\left\langle U_{n} f, g\right\rangle_{R^{n}}
$$

as required.

Recall that Hadamard introduced an inner product in the space $\mathfrak{F}$ of formal power series:

$$
(f * g)(x):=\sum_{k=0}^{\infty} c_{k} d_{k} x^{k} .
$$

This product can be retrieved as a special case of $\langle\cdot, \cdot\rangle_{R}$; namely,

$$
\left\langle\sum_{k=0}^{\infty} c_{k} x^{k}, \overline{\sum_{k=0}^{\infty} d_{k} x^{k}}\right\rangle_{R^{1 / 2}}=\sum_{k=0}^{\infty} c_{k} d_{k} R^{k} .
$$

Thus the Hadamard product is completely equivalent to our inner product. 


\section{Elementary properties of the operators $U_{n}$ and $V_{n}$}

In this section we describe elementary properties of the operators defined in (1.2) and (1.3).

THEOREM 3.1. Let $m, n \in \mathbb{N}$. Then the following hold.

(a) $U_{n} \circ U_{m}=U_{m} \circ U_{n}=U_{n m}$.

(b) $V_{n} \circ V_{m}=V_{m} \circ V_{n}=V_{n m}$.

(c) $U_{n} \circ V_{n}=$ Id.

(d) $U_{n} \circ V_{m}=V_{m} / \operatorname{gcd}(m, n) \circ U_{n / \operatorname{gcd}(m, n)}$. In particular, if $m$ and $n$ are relatively prime, then $U_{n}$ and $V_{m}$ commute.

PROOF. Let $f \in \mathfrak{F}$ be a formal power series with coefficients $c_{k}$. The first two properties follow directly from the observation that

$$
U_{n} U_{m} f(x)=U_{n} \sum_{k=0}^{\infty} c_{m k} x^{k}=\sum_{k=0}^{\infty} c_{n m k} x^{k}=U_{n m} f(x),
$$

and similarly for $V_{n} V_{m}$. To establish the third property observe that

$$
\begin{aligned}
U_{n} V_{n} f(x) & =U_{n} V_{n}\left(\sum_{k=0}^{\infty} c_{k} x^{k}\right)=U_{n}\left(\sum_{k=0}^{\infty} c_{k} x^{k n}\right) \\
& =\sum_{k=0}^{\infty} c_{k} x^{k}=f(x) .
\end{aligned}
$$

Finally,

$$
U_{n} \circ V_{m}\left(\sum_{k=0}^{\infty} c_{k} x^{k}\right)=U_{n}\left(\sum_{k=0}^{\infty} c_{k} x^{m k}\right) .
$$

To simplify this, let

$$
d_{k}= \begin{cases}c_{k / m} & \text { if } m \text { divides } k \\ 0 & \text { if } m \text { does not divide } k\end{cases}
$$

and write

$$
U_{n} \circ V_{m}\left(\sum_{k=0}^{\infty} c_{k} x^{k}\right)=U_{n}\left(\sum_{k=0}^{\infty} d_{k} x^{k}\right)=\sum_{k=0}^{\infty} d_{n k} x^{k} .
$$

Now observe that $m$ divides $k n$ if and only if $m / \operatorname{gcd}(m, n)$ divides $k$. Therefore, we define

$$
N=\frac{n}{\operatorname{gcd}(m, n)} \quad \text { and } \quad M=\frac{m}{\operatorname{gcd}(m, n)},
$$

and we obtain the following sum on $k$ :

$$
\begin{aligned}
\sum_{k} d_{n k} x^{k} & =\sum_{m \mid n k} d_{n k} x^{k}=\sum_{m \mid n k} c_{n k / m} x^{k} \\
& =\sum_{M \mid N k} c_{(N k) / M} x^{k}=\sum_{M \mid k} c_{N(k / M)} x^{k},
\end{aligned}
$$


where each sum is defined for $k$ from 0 to $\infty$, and subject to the constraints given. We now let $k=i M$, and sum over $i \geq 0$, and see that

$$
\sum_{M \mid k} c_{N(k / M)} x^{k}=\sum_{i=0}^{\infty} c_{i N} x^{i M}
$$

Now define $h_{i}=c_{i N}$, and obtain

$$
\sum_{i=0}^{\infty} h_{i} x^{i M}=V_{M}\left(\sum_{i=0}^{\infty} h_{i} x^{i}\right)=V_{M}\left(\sum_{i=0}^{\infty} c_{i N} x^{i}\right)=V_{M} \circ U_{N}\left(\sum_{i=0}^{\infty} c_{i} x^{i}\right),
$$

and we have established part (d).

We now present an alternate proof for Theorem 3.1. To do this, we must prove an intermediate result.

Lemma 3.2 (Associativity of $U$ and $V$ ). For all $k, j, m \in \mathbb{N}$,

$$
U_{k j} \circ V_{m}=U_{k} \circ\left(U_{j} \circ V_{m}\right) .
$$

PROOF. Let $f(z)=\sum_{n=0}^{\infty} a_{n} z^{n}$. For this proof, we will evaluate both operators and show they give the same result. First, for $U_{k j} \circ V_{m}$,

$$
\left(U_{k j} \circ V_{m}\right) f(z)=U_{k j} f\left(z^{m}\right)=U_{k j}\left(\sum_{n=0}^{\infty} a_{n} z^{m n}\right) .
$$

Now we let

$$
b_{i}= \begin{cases}a_{i / m} & \text { if } m \text { divides } i \\ 0 & \text { otherwise }\end{cases}
$$

so that we can write

$$
U_{k j}\left(\sum_{n=0}^{\infty} a_{n} z^{m n}\right)=U_{k j}\left(\sum_{i=0}^{\infty} b_{i} z^{i}\right)=\sum_{i=0}^{\infty} b_{(k j) i} z^{i} .
$$

Next, for $U_{k} \circ\left(U_{j} \circ V_{m}\right)$,

$$
U_{k} \circ\left(U_{j} \circ V_{m}\right)\left(\sum_{n=0}^{\infty} a_{n} z^{n}\right)=U_{k}\left(U_{j}\left(\sum_{n=0}^{\infty} a_{n} z^{m n}\right)\right)=U_{k}\left(\sum_{i=0}^{\infty} c_{i} z^{i}\right),
$$

where

$$
c_{i}= \begin{cases}a_{n} & \text { if } i j=m n \text { for } n \in \mathbb{N} \cup\{0\}, \\ 0 & \text { otherwise }\end{cases}
$$

Then

$$
U_{k}\left(\sum_{i=0}^{\infty} c_{i} z^{i}\right)=\sum_{i=0}^{\infty} c_{k i} z^{i} .
$$

We complete the proof by noting that $b_{(k j) i}=c_{k i}$. 
Alternative Proof of Theorem 3.1(d). We can write

$$
U_{n} \circ V_{m}=\left(U_{n / \operatorname{gcd}(m, n)} \circ U_{\operatorname{gcd}(m, n)}\right) \circ\left(V_{\operatorname{gcd}(m, n)} \circ V_{m / \operatorname{gcd}(m, n)}\right),
$$

by parts (a) and (b) of the theorem. Now, using associativity, we can write

$$
U_{n} \circ V_{m}=U_{n / \operatorname{gcd}(m, n)} \circ\left(U_{\operatorname{gcd}(m, n)} \circ V_{\operatorname{gcd}(m, n)}\right) \circ V_{m / \operatorname{gcd}(m, n)} .
$$

By part (c) of the theorem, we see that $U_{\operatorname{gcd}(m, n)} \circ V_{\operatorname{gcd}(m, n)}=I$, and we are left with $U_{n} \circ V_{m}=U_{m / \operatorname{gcd}(m, n)} \circ V_{\operatorname{gcd}(m, n)}$. Thus, part (d) of the theorem is proven.

\section{The action of $U_{n}$ on formal power series}

We now determine an expression for the action of the operator $U_{n}$ on formal power series where we allow the first few coefficients to vanish. This result will be employed in our study of spectral properties of $U_{n}$ acting on hypergeometric functions. For the rest of this section, all functions are assumed to be formal power series.

TheOrem 4.1. Let $j, n \in \mathbb{N}$. Then

$$
U_{n}\left(x^{j} \sum_{k=0}^{\infty} a_{k} x^{k}\right)= \begin{cases}x^{1+\lfloor j / n\rfloor} \sum_{k=0}^{\infty} a_{n(k+1-\{j / n\})} x^{k} & \text { if } n \text { does not divide } j, \\ x^{j / n} \sum_{k=0}^{\infty} a_{k n} x^{k} & \text { if } n \text { divides } j .\end{cases}
$$

PROOF. First observe that

$$
U_{n}\left(x^{j} \sum_{k=0}^{\infty} a_{k} x^{k}\right)=U_{n}\left(\sum_{k=0}^{\infty} a_{k} x^{k+j}\right)=U_{n}\left(\sum_{k=j}^{\infty} a_{k-j} x^{k}\right),
$$

and define

$$
b_{k}= \begin{cases}0 & \text { if } 0 \leq k<j \\ a_{k-j} & \text { if } k \geq j\end{cases}
$$

then we can write

$$
U_{n}\left(x^{j} \sum_{k=0}^{\infty} a_{k} x^{k}\right)=U_{n}\left(\sum_{k=0}^{\infty} b_{k} x^{k}\right)=\sum_{k=0}^{\infty} b_{k n} x^{k} .
$$

The discussion is divided into two cases, according to whether $n$ divides $j$ or not.

Case 1: $n$ does not divide $j$. In this case, the restriction $k n \geq j$ in (4.1) is equivalent to $k \geq\lfloor j / n\rfloor+1$. Thus,

$$
\begin{aligned}
\sum_{k=0}^{\infty} b_{k n} x^{k} & =\sum_{k=0}^{\lfloor j / n\rfloor} b_{k n} x^{k}+\sum_{k=\lfloor j / n\rfloor+1}^{\infty} b_{k n} x^{k} \\
& =\sum_{k=\lfloor j / n\rfloor+1}^{\infty} a_{k n-j} x^{k}
\end{aligned}
$$


Now let $i=k-\lfloor j / n\rfloor-1$, to obtain

$$
\sum_{k=0}^{\infty} b_{k n} x^{k}=x^{\lfloor j / n\rfloor+1} \sum_{i=0}^{\infty} a_{n i+n\lfloor j / n\rfloor+n-j} x^{i} .
$$

Now use the formula $j / n=\lfloor j / n\rfloor+\{j / n\}$ to obtain

$$
U_{n}\left(x^{j} \sum_{k=0}^{\infty} a_{k} x^{k}\right)=x^{\lfloor j / n\rfloor+1} \sum_{i=0}^{\infty} a_{n(i+1-\{j / n\})} x^{i} .
$$

This is the result when $n$ does not divide $j$.

Case $2: n$ divides $j$. In this case, the condition $k n \geq j$ is equivalent to $k \geq j / n=$ $\lfloor j / n\rfloor$, and

$$
\begin{aligned}
\sum_{k=0}^{\infty} b_{k n} x^{k} & =\sum_{k=\lfloor j / n\rfloor}^{\infty} b_{k n} x^{k} \\
& =\sum_{k=\lfloor j / n\rfloor}^{\infty} a_{k n-j} x^{k} \\
& =x^{\lfloor j / n\rfloor} \sum_{i=0}^{\infty} a_{n i} x^{i}
\end{aligned}
$$

so that

$$
U_{n}\left(x^{j} \sum_{k=0}^{\infty} a_{k} x^{k}\right)=x^{\lfloor j / n\rfloor} \sum_{\nu \geq 0} a_{n \nu} x^{\nu} .
$$

This concludes the proof.

The expressions for $U_{n}$ are now used to derive some elementary properties of its eigenfunctions on the space of formal power series.

PROPOSITION 4.2. Assume that $U_{n}$ has an eigenfunction of the form

$$
f(x)=x^{j} \sum_{k=0}^{\infty} a_{k} x^{k}
$$

with eigenvalue $\lambda$. If $n$ divides $j$, then $j=0$ and $\lambda=1$. If $n$ does not divide $j$, then $j=1$.

PROOF. Assume that $n$ divides $j$ and match the leading order terms of $f$ and $U_{n} f$. Theorem 4.1 shows that $x^{j}=x^{j / n}$, which yields $j=0$. Now compare the constant terms in the eigenvalue equations to see that $\lambda=1$. In the case where $n$ does not divide $j$, the same comparison yields

$$
j=1+\lfloor j / n\rfloor \text {. }
$$

This implies that $j=1$. Indeed, let $j=\alpha n+\beta$ where $0<\beta<n$. Then $j=1+\alpha$, and this yields

$$
1-\beta=\alpha(n-1)
$$


It follows that $\beta=1$ and $\alpha=0$, otherwise the two sides of (4.2) have different signs. We conclude that $j=1+\alpha=1$.

Hence even for a formal power series $f$, we see that the assumption that $f$ is an eigenfunction of the Hecke operator $U_{n}$ imposes the restriction that $f$ can only vanish to order zero or one.

For the sake of completeness, we describe the trivial eigenfunctions of the composition of operators $U_{n} \circ V_{n}$ and $V_{n} \circ U_{n}$. Theorem 3.1 shows that $U_{n} \circ V_{n}$ is the identity. The next result describes the composition $V_{n} \circ U_{n}$.

THEOREM 4.3. The only eigenvalue of $V_{n} \circ U_{n}$ is 1 . Moreover, given any formal power series $f(x)=\sum_{k=0}^{\infty} b_{k} x^{k}$, the function $g(x)=\sum_{k=0}^{\infty} a_{k} x^{k}$, where

$$
a_{k}= \begin{cases}b_{k} & \text { if } n \text { divides } k \\ 0 & \text { if } n \text { does not divide } k\end{cases}
$$

is an eigenfunction of $V_{n} \circ U_{n}$, with eigenvalue 1 .

PROOF. We consider the identity

$$
\left(V_{n} \circ U_{n}\right)\left(\sum_{k=0}^{\infty} a_{k} x^{k}\right)=\sum_{k=0}^{\infty} a_{k n} x^{k}
$$

and we are done.

\section{The hypergeometric functions}

In this section we use Theorem 4.1 to describe the action of $U_{n}$ on the set $\mathfrak{H}$ of all hypergeometric functions. We recall that a hypergeometric function is defined by the power series

$$
{ }_{p} F_{q}(\mathbf{a}, \mathbf{b} ; x):=\sum_{k=0}^{\infty} \frac{\left(a_{1}\right)_{k}\left(a_{2}\right)_{k} \cdots\left(a_{p}\right)_{k}}{\left(b_{1}\right)_{k}\left(b_{2}\right)_{k} \cdots\left(b_{q}\right)_{k}} \frac{x^{k}}{k !},
$$

where $\mathbf{a}:=\left(a_{1}, a_{2}, \ldots, a_{p}\right)$ and $\mathbf{b}:=\left(b_{1}, b_{2}, \ldots, b_{q}\right)$ are the parameters of ${ }_{p} F_{q}$. These parameters are nonzero complex numbers. We begin by stating explicitly the action of $U_{n}$ on $\mathfrak{F}_{(p, q)}^{j}$ as the main result of this section.

THEOREM 5.1. Let $j, n \in \mathbb{N}$. The action of $U_{n}$ on the class $\mathfrak{F}_{(p, q)}^{j}$, that is, on functions of the form

$$
f_{p, q, j}=x^{j}{ }_{p} F_{q}(\mathbf{a}, \mathbf{b} ; x)
$$

is characterized as follows.

If $n$ divides $j$, then

$$
U_{n}\left(x^{j} \sum_{k=0}^{\infty} \frac{\left(a_{1}\right)_{k} \cdots\left(a_{p}\right)_{k}}{\left(b_{1}\right)_{k} \cdots\left(b_{q+1}\right)_{k}} x^{k}\right)=x^{j / n} \sum_{k=0}^{\infty} \frac{\left(c_{1}\right)_{k}\left(c_{2}\right)_{k} \cdots\left(c_{n p}\right)_{k}}{\left(d_{1}\right)_{k}\left(d_{2}\right)_{k} \cdots\left(d_{n(q+1)-1}\right)_{k}} \frac{x_{1}^{k}}{k !},
$$


where the parameters $\mathbf{c}$ and $\mathbf{d}$ are defined by

$$
\begin{array}{cl}
c_{i n+l}=\frac{a_{i+1}+l-1}{n} & \text { if } 0 \leq i \leq p-1,1 \leq l \leq n, \\
d_{i n+l}=\frac{b_{i+1}+l-1}{n} & \text { if } 0 \leq i \leq q, 1 \leq l \leq n,
\end{array}
$$

and $j_{1}=j / n$. The new variable is $x_{1}=n^{n(p-q-1)} x$.

If $n$ does not divide $j$, then

$$
U_{n}\left(x^{j} \sum_{k=0}^{\infty} \frac{\left(a_{1}\right)_{k} \cdots\left(a_{p}\right)_{k}}{\left(b_{1}\right)_{k} \cdots\left(b_{q+1}\right)_{k}} x^{k}\right)=x^{1+\lfloor j / n\rfloor} \sum_{k=0}^{\infty} \frac{\left(c_{1}\right)_{k} \cdots\left(c_{p n}\right)_{k}}{\left(d_{1}\right)_{k} \cdots\left(d_{(q+1) n-1}\right)_{k}} \frac{x_{1}^{k}}{k !},
$$

where the parameters $\mathbf{c}$ and $\mathbf{d}$ are now defined by

$$
\begin{array}{cl}
c_{i n+l}=\frac{a_{i+1}+r+l}{n} & \text { if } 0 \leq i \leq p-1,1 \leq l \leq n, \\
d_{i n+l}=\frac{b_{i+1}+r+l}{n} & \text { if } 0 \leq i \leq q, 1 \leq l \leq n,
\end{array}
$$

and $j_{2}=1+\lfloor j / n\rfloor$ and $r=n(1-\{j / n\})-1$. The new variable $x_{1}$ is defined as above.

Before proving this theorem, we first need to state some intermediate results. The next lemma allows for a simplification of the ascending factorial function on an arithmetic progression of indices.

LemMa 5.2. Let $k, n \in \mathbb{N}$ and $a \in \mathbb{R}$. Then

$$
(a)_{k n}=n^{k n} \prod_{j=0}^{n-1}\left(\frac{a+j}{n}\right)_{k}
$$

PROOF. Start with the observation that

$$
(a)_{k n}=\prod_{i=0}^{k n-1}(a+i)=n^{k n} \prod_{i=0}^{k n-1}\left(\frac{a}{n}+\frac{i}{n}\right)
$$

and then collect terms according to their classes modulo $n$.

In order to evaluate

$$
U_{n}\left(x^{j}{ }_{p} F_{q}(\mathbf{a}, \mathbf{b})\right)=U_{n}\left(x^{j} \sum_{k=0}^{\infty} \frac{\left(a_{1}\right)_{k}\left(a_{2}\right)_{k} \cdots\left(a_{p}\right)_{k}}{\left(b_{1}\right)_{k}\left(b_{2}\right)_{k} \cdots\left(b_{q}\right)_{k}\left(b_{q+1}\right)_{k}} x^{k}\right),
$$

where we used the fact that $k !=(1)_{k}$ and defined $b_{q+1}=1$, we observe that, by Theorem 4.1, the discussion should be divided into two cases according to whether or not $n$ divides $j$. 
Case 1: $n$ divides $j$. In this case, Theorem 4.1 yields

$$
U_{n}\left(x^{j}{ }_{p} F_{q}(\mathbf{a}, \mathbf{b})\right)=x^{j / n} \sum_{k=0}^{\infty} \frac{\left(a_{1}\right)_{k n} \cdots\left(a_{p}\right)_{k n}}{\left(b_{1}\right)_{k n} \cdots\left(b_{q+1}\right)_{k n}} x^{k},
$$

and using Lemma 5.2 we can write this as

$$
\begin{aligned}
U_{n}\left(x^{j}{ }_{p} F_{q}(\mathbf{a}, \mathbf{b})\right)=x^{j / n} & \sum_{k=0}^{\infty} n^{(p-q-1) k n}\left(\prod_{j=1}^{p} \prod_{i=0}^{n-1}\left(\frac{a_{j}+i}{n}\right)_{k}\right) \\
& \times\left(\prod_{j=1}^{q+1} \prod_{i=0}^{n-1}\left(\frac{b_{j}+i}{n}\right)_{k}\right)^{-1} x^{k} .
\end{aligned}
$$

Now recall that $b_{q+1}=1$, so the $d$-parameters when $i=q$ from the definition (5.2) are $1 / n, 2 / n, \ldots,(n-1) / n, 1$. The total number of $d$-parameters is now reduced by 1 , in order to write the result in the canonical hypergeometric form:

$$
U_{n}\left(x_{p}^{j} F_{q}(\mathbf{a}, \mathbf{b})\right)=x^{j / n} \sum_{k=0}^{\infty} n^{(p-q-1) k n} \frac{\left(c_{1}\right)_{k}\left(c_{2}\right)_{k} \cdots\left(c_{n p}\right)_{k}}{\left(d_{1}\right)_{k}\left(d_{2}\right)_{k} \cdots\left(d_{n(q+1)-1}\right)_{k}} \frac{x^{k}}{k !} .
$$

LEMMA 5.3. The parameters $\mathbf{a}, \mathbf{b}, \mathbf{c}$ and $\mathbf{d}$ satisfy

$$
\sum_{i=1}^{n p} c_{i}-\sum_{i=1}^{n(q+1)-1} d_{i}=\sum_{i=1}^{p} a_{i}-\sum_{i=1}^{q} b_{i}+\frac{(n-1)}{2}(p-q-1) .
$$

PROOF. The new parameters are

$$
\frac{a_{1}}{n}, \frac{a_{1}+1}{n}, \ldots, \frac{a_{1}+n-1}{n}, \frac{a_{p}}{n}, \ldots, \frac{a_{p}+n-1}{n}
$$

and

$$
\frac{b_{1}}{n}, \frac{b_{1}+1}{n}, \ldots, \frac{b_{1}+n-1}{n}, \frac{b_{q+1}}{n}=\frac{1}{n}, \ldots, \frac{b_{q+1}+n-2}{n}=\frac{n-1}{n},
$$

and the identity is now easy to check.

COROLlaRY 5.4. If $p=q+1$, then $\sum_{i=1}^{p} a_{i}-\sum_{i=1}^{q} b_{i}$ is preserved under the action of $U_{n}$.

Case 2: $n$ does not divide $j$. In this case, Theorem 4.1 gives

$$
U_{n}\left(x^{j} \sum_{k=0}^{\infty} \frac{\left(a_{1}\right)_{k} \cdots\left(a_{p}\right)_{k}}{\left(b_{1}\right)_{k} \cdots\left(b_{q+1}\right)_{k}} x^{k}\right)=x^{1+\lfloor j / n\rfloor} \sum_{k=0}^{\infty} \frac{\left(a_{1}\right)_{N} \cdots\left(a_{p}\right)_{N}}{\left(b_{1}\right)_{N} \cdots\left(b_{q+1}\right)_{N}} x^{k},
$$

where $b_{q+1}=1$ and we define $N=n(k+1-\{j / n\})$. Observe that $0<\{j / n\}<1$, and thus $n k<N<n(k+1)$. The next result simplifies the Pochhammer symbols. 
Lemma 5.5. Let $a \in \mathbb{C}$ and $j, n \in \mathbb{N}$ where $j$ is not divisible by $n$. Define $N=$ $n(k+1-\{j / n\})$ and $r=n(1-\{j / n\})-1$. Then

$$
(a)_{N}=n^{n k}(a)_{r+1} \prod_{i=r+1}^{r+n}\left(\frac{a+i}{n}\right)_{k} .
$$

PROOF. We begin by recalling that

$$
\begin{aligned}
(a)_{N} & =a(a+1)(a+2) \cdots(a+N-1) \\
& =n^{N}\left[\frac{a}{n}\left(\frac{a}{n}+\frac{1}{n}\right) \cdots\left(\frac{a}{n}+\frac{N-1}{n}\right)\right] .
\end{aligned}
$$

We now regroup terms modulo $n$ as follows:

$$
\begin{aligned}
n^{-N}(a)_{N}=\left(\frac{a}{n}\right) & \cdot\left(\frac{a}{n}+1\right) \cdots\left(\frac{a}{n}+k-1\right) \\
& \times\left(\frac{a}{n}+\frac{1}{n}\right) \cdot\left(\frac{a}{n}+\frac{1}{n}+1\right) \cdots\left(\frac{a}{n}+\frac{1}{n}+k-1\right) \\
& \cdots\left(\frac{a}{n}+\frac{n-1}{n}\right) \cdot\left(\frac{a}{n}+\frac{n-1}{n}+1\right) \cdots\left(\frac{a}{n}+\frac{n-1}{n}+k-1\right) \\
& \times\left\{\left(\frac{a}{n}+k\right) \cdot\left(\frac{a}{n}+\frac{1}{n}+k\right) \cdots\left(\frac{a}{n}+\frac{r}{n}+k\right)\right\} .
\end{aligned}
$$

The last factor appears because $n$ does not divide $j$. Therefore

$$
(a)_{N}=n^{N} \prod_{i=0}^{n-1}\left(\frac{a+i}{n}\right)_{k} \times \prod_{i=0}^{r}\left(\frac{a+i}{n}+k\right)
$$

where the second product does not involve the Pochhammer symbol. Now employ the relation

$$
k+c=c \frac{(c+1)_{k}}{(c)_{k}}
$$

to write

$$
(a)_{N}=n^{N} \prod_{i=0}^{n-1}\left(\frac{a+i}{n}\right)_{k} \prod_{i=0}^{r}\left(\frac{a+i}{n}\right) \cdot \prod_{i=0}^{r}\left(\frac{a+i}{n}+1\right)_{k} / \prod_{i=0}^{r}\left(\frac{a+i}{n}\right)_{k}
$$

This expression reduces to the stated formula.

The transformation above yields

$$
U_{n}\left(x^{j} \sum_{k=0}^{\infty} \frac{\left(a_{1}\right)_{k} \cdots\left(a_{p}\right)_{k}}{\left(b_{1}\right)_{k} \cdots\left(b_{q+1}\right)_{k}} x^{k}\right)=x^{1+\lfloor j / n\rfloor} \sum_{k=0}^{\infty} n^{n k(p-q-1)} \frac{\left(c_{1}\right)_{k} \cdots\left(c_{p n}\right)_{k}}{\left(d_{1}\right)_{k} \cdots\left(d_{(q+1) n}\right)_{k}} x^{k} .
$$

The special case where $p=q+1$ provides a simpler situation, in which the coefficient $n^{n k(p-q-1)}$ does not appear in the resulting series. 
THEOREM 5.6. Let $j, n \in \mathbb{N}$ and assume that $p=q+1$.

If $n$ divides $j$, then

$$
U_{n}\left(x^{j}{ }_{p} F_{q}(\mathbf{a}, \mathbf{b} ; x)\right)=x^{j / n}{ }_{n p} F_{n p-1}(\mathbf{c}, \mathbf{d} ; x),
$$

where $\mathbf{c}$ and $\mathbf{d}$ are defined in (5.1).

If $n$ does not divide $j$, then

$$
U_{n}\left(x_{p}^{j} F_{q}(\mathbf{a}, \mathbf{b} ; x)\right)=x^{1+\lfloor j / n\rfloor}{ }_{n p} F_{n p-1}(\mathbf{c}, \mathbf{d} ; x),
$$

where $\mathbf{c}$ and $\mathbf{d}$ are defined in (5.2).

\section{The eigenvalue equation}

In this section we focus on the spectral properties of the operator $U_{n}$, as the spectral properties of the operators $V_{n}$ are trivial. It is here that we encounter more subtle ideas. We describe first the eigenfunctions of the operator $U_{n}$ of the form $x^{j}{ }_{p} F_{q}(\mathbf{a}, \mathbf{b} ; x)$. That is, we look for parameters $p, q \in \mathbb{N}$ and complex numbers

$$
a_{1}, a_{2}, \ldots, a_{p} ; b_{1}, b_{2}, \ldots, b_{q}
$$

such that, with $\mathbf{a}=\left(a_{1}, \ldots, a_{p}\right)$ and $\mathbf{b}=\left(b_{1}, \ldots, b_{q}\right)$,

$$
U_{n}\left(x^{j}{ }_{p} F_{q}(\mathbf{a}, \mathbf{b} ; x)\right)=\lambda x^{j}{ }_{p} F_{q}(\mathbf{a}, \mathbf{b} ; x) .
$$

The results from Theorem 5.1 showed that the action of $U_{n}$ on $x_{p}^{j} F_{q}$ depends on whether or not $n$ divides $j$, which by Proposition 4.2 reduces to the cases in which $j=0$ and $j=1$ when considering eigenfunctions of $U_{n}$.

Case 1: $j=0$. Under this condition, we show that the eigenfunction reduces to a rational function.

Lemma 6.1. Assume that $n$ divides $j$ and that (6.1) has a nontrivial solution. Then, for all $k \in \mathbb{N}$,

$$
\begin{aligned}
& \prod_{j=1}^{p} \prod_{i=0}^{n-1}\left(a_{j}+n k+i\right) \times \prod_{j=1}^{q+1}\left(b_{j}+k\right) \\
& \quad=\prod_{j=1}^{p}\left(a_{j}+j\right) \times \prod_{j=1}^{q+1} \prod_{i=0}^{n-1}\left(b_{j}+n k+i\right) .
\end{aligned}
$$

PROOF. Comparing terms of the equation $U_{n} f=f$ yields

$$
\frac{\left(a_{1}\right)_{n k}\left(a_{2}\right)_{n k} \cdots\left(a_{p}\right)_{n k}}{\left(b_{1}\right)_{n k}\left(b_{2}\right)_{n k} \cdots\left(b_{q+1}\right)_{n k}}=\frac{\left(a_{1}\right)_{k}\left(a_{2}\right)_{k} \cdots\left(a_{p}\right)_{k}}{\left(b_{1}\right)_{k}\left(b_{2}\right)_{k} \cdots\left(b_{q+1}\right)_{k}} .
$$

Replace $k$ by $k+1$, divide the two equations and use the formulae

$$
\frac{(a)_{k+1}}{(a)_{k}}=a+k \quad \text { and } \quad \frac{(a)_{n(k+1)}}{(a)_{k}}=(a+n k)(a+n k+1) \cdots(a+n k+n-1)
$$

to produce the result. 
Lemma 6.2. Assume that $n$ divides $j$ and that (6.1) has a nontrivial solution. Then $p=q+1$.

PROOF. Comparing the degrees of the left- and right-hand side of (6.2) gives the equality $p n+q+1=p+n(q+1)$.

Proposition 6.3. Assume that $n$ divides $j$ and that (6.1) has a nontrivial solution. Then

$$
\left\{a_{i}, \frac{b_{i}}{n}, \frac{b_{i}+1}{n}, \ldots, \frac{b_{i}+n-1}{n}\right\}_{i=1}^{p}=\left\{b_{i}, \frac{a_{i}}{n}, \frac{a_{i}+1}{n}, \ldots, \frac{a_{i}+n-1}{n}\right\}_{i=1}^{p} .
$$

PROOF. The roots of the left- and right-hand side of (6.2) must match.

We now show that the results of this proposition imply that the parameters must match: $a_{i}=b_{i}$ for all indices.

PROPOSITION 6.4. Assume that $n$ divides $j$ and that (6.1) has a nontrivial solution. Then, for any $k \in \mathbb{N}$,

$$
\sum_{i=1}^{p} a_{i}^{k}=\sum_{i=1}^{p} b_{i}^{k}
$$

Proof. Proof is by induction on $k$. The case where $k=1$ comes from matching the coefficients of the next to leading order in $k$. Indeed, this matching yields

$$
\sum_{i=1}^{p} a_{i}+\sum_{j=1}^{n-1} \sum_{i=1}^{p}\left(b_{i}+j\right)=\sum_{i=1}^{p} b_{i}+\sum_{j=1}^{n-1} \sum_{i=1}^{p}\left(a_{i}+j\right)
$$

and the case where $k=1$ holds. In order to check it for $k=2$, add the squares of the elements in Proposition 6.3 to obtain, from the left-hand side, the expression

$$
\begin{aligned}
& \sum_{i=1}^{p} a_{i}^{2}+\frac{1}{n^{2}} \sum_{i=1}^{p} \sum_{j=0}^{n-1}\left(b_{i}^{2}+2 j b_{i}+j^{2}\right) \\
& \quad=\sum_{i=1}^{p} a_{i}^{2}+\frac{1}{n} \sum_{i=1}^{p} b_{i}^{2}+\frac{2}{n^{2}} \sum_{i=1}^{p} b_{i} \times \sum_{j=0}^{n-1} j+\frac{p}{n^{2}} \sum_{j=0}^{n-1} j^{2}
\end{aligned}
$$

Matching with the corresponding expression from the right-hand side and using the statement for $k=1$ yields

$$
\sum_{i=1}^{p} a_{i}^{2}=\sum_{i=1}^{p} b_{i}^{2}
$$

The higher moments can be established along these lines. 
Proposition 6.5. Assume that two sets $\left\{a_{j}: 1 \leq j \leq p\right\}$ and $\left\{b_{j}: 1 \leq j \leq p\right\}$ of complex numbers satisfy

$$
\sum_{i=1}^{p} a_{i}^{k}=\sum_{i=1}^{p} b_{i}^{k}
$$

for every $k \in \mathbb{N}$. Then, after a possible rearrangement of the order of the terms of one of these sets, $a_{i}=b_{i}$ for all $i$.

Proof. For $\mathbf{a}, \mathbf{b} \in \mathbb{C}^{p}$ and $N \in \mathbb{N}$, let

$$
\mu_{N}(\mathbf{a})=\sum_{i=1}^{p} a_{i}^{N}
$$

and let

$$
f_{\mathbf{a}}(t)=\sum_{j=0}^{\infty} \mu_{j}(\mathbf{a}) \frac{t^{j}}{j !} \quad \text { and } \quad f_{\mathbf{b}}(t)=\sum_{j=0}^{\infty} \mu_{j}(\mathbf{b}) \frac{t^{j}}{j !}
$$

be the generating functions of $\mu_{N}(\mathbf{a})$ and $\mu_{N}(\mathbf{b})$, respectively. Now assume that $f_{\mathbf{a}}=f_{\mathbf{b}}$, that is,

$$
\sum_{j=0}^{\infty} \mu_{j}(\mathbf{a}) \frac{t^{j}}{j !}=\sum_{j=0}^{\infty} \mu_{j}(\mathbf{b}) \frac{t^{j}}{j !} .
$$

Expanding further gives

$$
\sum_{j=0}^{\infty} \sum_{i=1}^{p} a_{i}^{j} \frac{t^{j}}{j !}=\sum_{j=0}^{\infty} \sum_{i=1}^{p} b_{i}^{j} \frac{t^{j}}{j !}
$$

Since $\mu_{j}$ is defined as a sum of finite terms, we can change the order of summation:

$$
\sum_{i=1}^{p} \sum_{j=0}^{\infty} a_{i}^{j} \frac{t^{j}}{j !}=\sum_{i=1}^{p} \sum_{j=0}^{\infty} b_{i}^{j} \frac{t^{j}}{j !}
$$

This yields

$$
e^{a_{1} t}+e^{a_{2} t}+\cdots+e^{a_{p} t}=e^{b_{1} t}+e^{b_{2} t}+\cdots+e^{b_{p} t} .
$$

Suppose first that $a_{i}, b_{i} \in \mathbb{R}$ and order them as

$$
a_{1} \leq a_{2} \leq \cdots \leq a_{p} \quad \text { and } \quad b_{1} \leq b_{2} \leq \cdots \leq b_{p} .
$$

Eliminate from (6.3) all the terms for which the $a$ s and $b$ s match, to assume that $a_{1}<b_{1}$. Then

$$
1+e^{\left(a_{2}-a_{1}\right) t}+\cdots+e^{\left(a_{p}-a_{1}\right) t}=e^{\left(b_{1}-a_{1}\right) t}+e^{\left(b_{2}-a_{1}\right) t}+\cdots+e^{\left(b_{p}-a_{1}\right) t} .
$$

Finally, let $t \rightarrow-\infty$ to get a contradiction. 
We summarize the previous discussion in the following theorem.

THEOREM 6.6. Suppose there exists an eigenfunction of $U_{n}$ of the form

$$
f(x)=\sum_{k=0}^{\infty} \frac{\left(a_{1}\right)_{k}\left(a_{2}\right)_{k} \cdots\left(a_{p}\right)_{k}}{\left(b_{1}\right)_{k}\left(b_{2}\right)_{k} \cdots\left(b_{q+1}\right)_{k}} x^{k},
$$

corresponding to the eigenvalue $\lambda$ (this is the case where $j=0$ in (6.1)). Then $\lambda=1, p=q+1$ and $a_{i}=b_{i}$ for all $i$. Therefore $f(x)=1 /(1-x)$.

Proposition 4.2 shows that the only possible values for $j$ are 0 and 1 . The rest of the section treats the case where $j=1$.

Case 2: $j=1$. Under this condition we show that the spectrum of the operator $U_{n}$ is the set $\left\{n^{i}: i \in \mathbb{Z}\right\}$. The corresponding eigenfunctions are the polylogarithm functions

$$
\operatorname{PolyLog}_{i}(x):=\sum_{k=1}^{\infty} k^{i} x^{k},
$$

corresponding to the eigenvalue $n^{i}$ when $i$ is negative, and the eigenfunctions

$$
\left(x \frac{d}{d x}\right)^{i}\left(\frac{1}{1-x}\right)
$$

corresponding to the eigenvalue $n^{i}$ when $i$ is nonnegative.

EXAMPLE 6.7. The dilogarithm function

$$
\operatorname{Li}_{2}(x):=\sum_{k=1}^{\infty} \frac{x^{k}}{k^{2}}
$$

satisfies

$$
U_{n}\left(\operatorname{Li}_{2}(x)\right)=\frac{1}{n^{2}} \operatorname{Li}_{2}(x) .
$$

Therefore $1 / n^{2} \in \operatorname{Spec}\left(U_{n}\right)$. The dilogarithm function admits the hypergeometric representation

$$
\operatorname{Li}_{2}(x)=x_{3} F_{2}\left(\mathbf{1}_{3}, \mathbf{2}_{2} ; x\right),
$$

where $\mathbf{1}_{3}$ stands for $(1,1,1)$ and $\mathbf{2}_{2}$ stands for $(2,2)$.

We now explore properties of eigenfunctions of the operator $U_{n}$.

PROPOSITION 6.8. Assume that (6.1) has a nontrivial solution for which $j=1$. Then, for all $k \in \mathbb{N}$,

$$
\prod_{j=1}^{p}\left(a_{j}+k-1\right) \cdot \prod_{j=1}^{q+1} \prod_{i=-1}^{n-2}\left(b_{j}+n k+i\right)=\prod_{j=1}^{q+1}\left(b_{j}+k-1\right) \cdot \prod_{j=1}^{p} \prod_{i=-1}^{n-2}\left(a_{j}+n k+i\right) .
$$


PROOF. Assume that the eigenfunction has the form

$$
f(x)=x \sum_{k=0}^{\infty} c_{k} x^{k}
$$

Comparing coefficients in the equation $U_{n} f=\lambda f$ gives

$$
c_{n k-1}=\lambda c_{k-1} \text {. }
$$

Replacing the standard hypergeometric type yields

$$
\frac{\left(a_{1}\right)_{n k-1} \cdots\left(a_{p}\right)_{n k-1}}{\left(b_{1}\right)_{n k-1} \cdots\left(b_{q+1}\right)_{n k-1}}=\lambda \frac{\left(a_{1}\right)_{k-1} \cdots\left(a_{p}\right)_{k-1}}{\left(b_{1}\right)_{k-1} \cdots\left(b_{q+1}\right)_{k-1}} .
$$

Replace $k$ by $k+1$ and divide the two corresponding equations to obtain the result.

LEMMA 6.9. Assume that $j=1$ and that (6.1) has a nontrivial solution of the form

$$
f(x)=x \sum_{k=0}^{\infty} \frac{\left(a_{1}\right)_{k} \cdots\left(a_{p}\right)_{k}}{\left(b_{1}\right)_{k} \cdots\left(b_{q+1}\right)_{k}} x^{k} .
$$

Then $p=q+1$.

PROOF. Compare the degrees on both sides of the polynomial in Proposition 6.8.

Proposition 6.10. Assume that

$$
f(x)=x \sum_{k=0}^{\infty} \frac{\left(a_{1}\right)_{k} \cdots\left(a_{p}\right)_{k}}{\left(b_{1}\right)_{k} \cdots\left(b_{p}\right)_{k}} x^{k}
$$

is an eigenfunction for $U_{n}$. Then

$$
\begin{aligned}
& \bigcup_{i=1}^{p}\left\{a_{i}-1, \frac{b_{i}-1}{n}, \frac{b_{i}}{n}, \ldots, \frac{b_{i}+n-2}{n}\right\} \\
& =\bigcup_{i=1}^{p}\left\{b_{i}-1, \frac{a_{i}-1}{n}, \frac{a_{i}}{n}, \ldots, \frac{a_{i}+n-2}{n}\right\} .
\end{aligned}
$$

PROOF. These are the roots of both sides of the polynomial in Proposition 6.8.

We now show that this equality of sets imposes severe restrictions on the eigenvalues and eigenfunctions of the operator $U_{n}$. We discuss first the eigenvalues.

Proposition 6.11. Assume that

$$
f(x)=x \sum_{k=0}^{\infty} \frac{\left(a_{1}\right)_{k} \cdots\left(a_{p}\right)_{k}}{\left(b_{1}\right)_{k} \cdots\left(b_{p}\right)_{k}} x^{k}
$$

is an eigenfunction for $U_{n}$. Let

$$
\gamma_{a}:=\left|\left\{i \in\{1,2, \ldots, p\}: a_{i}=1\right\}\right|,
$$

and

$$
\gamma_{b}:=\left|\left\{i \in\{1,2, \ldots, p\}: b_{i}=1\right\}\right|
$$


Then

$$
n^{\gamma_{a}}\left(a_{1}\right)_{n-1}\left(a_{2}\right)_{n-1} \cdots\left(a_{p}\right)_{n-1}=n^{\gamma_{b}}\left(b_{1}\right)_{n-1}\left(b_{2}\right)_{n-1} \cdots\left(b_{p}\right)_{n-1} .
$$

PROOF. Consider the product of all the nonzero terms on the left-hand side of Proposition 6.10. The removal of the zero terms, corresponding to those $a_{i}$ equal to 1 , carries with it the removal of a power of $n$.

EXAMPLE 6.12. In Example 6.7 we see that $\gamma_{a}=3$ and $\gamma_{b}=1$. Thus (6.5) states correctly that

$$
n^{3} \times(1)_{n-1}(1)_{n-1}(1)_{n-1}=n \times(2)_{n-1}(2)_{n-1}(1)_{n-1} .
$$

TheOrem 6.13. Assume that $j=1$ and

$$
f(x)=x \sum_{k=0}^{\infty} \frac{\left(a_{1}\right)_{k} \cdots\left(a_{p}\right)_{k}}{\left(b_{1}\right)_{k} \cdots\left(b_{p}\right)_{k}}
$$

is an eigenfunction for $U_{n}$ with eigenvalue $\lambda$. Then

$$
\lambda=n^{\gamma_{b}-\gamma_{a}}
$$

where $\gamma_{a}$ and $\gamma_{b}$ are defined in (6.5).

PROOF. Put $k=1$ in the relation (6.4) to obtain

$$
\lambda=\frac{\left(a_{1}\right)_{n-1} \cdots\left(a_{p}\right)_{n-1}}{\left(b_{1}\right)_{n-1} \cdots\left(b_{q+1}\right)_{n-1}} .
$$

Now use (6.5) to conclude.

The result above shows that the spectrum of $U_{n}$ satisfies

$$
\operatorname{Spec}\left(U_{n}\right) \subseteq\left\{n^{a}: a \in \mathbb{Z}\right\} .
$$

The example below shows that actually equality holds.

EXAmple 6.14. Let $i \in \mathbb{Z}$. Then the hypergeometric series

$$
f_{i}(x):=\sum_{k=1}^{\infty} k^{i} x^{k},
$$

is an eigenfunction of $U_{n}$, with eigenvalue $n^{i}$. The dilogarithm corresponds to the case where $i=-2$.

THEOREM 6.15. The spectrum of $U_{n}$ is the set $\left\{n^{i}: i \in \mathbb{Z}\right\}$.

We now discuss the eigenfunctions of $U_{n}$, starting with the sets

$$
\begin{aligned}
& \bigcup_{i=1}^{p}\left\{a_{i}-1, \frac{b_{i}-1}{n}, \frac{b_{i}}{n}, \ldots, \frac{b_{i}+n-2}{n}\right\} \\
& =\bigcup_{i=1}^{p}\left\{b_{i}-1, \frac{a_{i}-1}{n}, \frac{a_{i}}{n}, \ldots, \frac{a_{i}+n-2}{n}\right\},
\end{aligned}
$$


that appeared in Proposition 6.10. Recall that $\left\{a_{i}, b_{i}\right\}$ are the parameters of the eigenfunction

$$
f(x)=x \sum_{k=0}^{\infty} \frac{\left(a_{1}\right)_{k} \cdots\left(a_{p}\right)_{k}}{\left(b_{1}\right)_{k} \cdots\left(b_{q+1}\right)_{k}} x^{k} .
$$

We may assume that $a_{i} \neq b_{j}$, otherwise we just cancel the equal terms $\left(a_{i}\right)_{k}$ and $\left(b_{j}\right)_{k}$. Observe that if we let $c_{i}=a_{i}-1$ and $d_{i}=b_{i}-1$, the basic set identity in Case 2 of Proposition 6.10 becomes the basic set identity of Case 1 , with $c_{i}$ instead of $a_{i}$ and $d_{i}$ instead of $b_{i}$. The reason why one cannot deduce $a_{i}=b_{i}$ is that some of the $a_{i}$ in Case 2 might be 1 , and the corresponding $c_{i}$ would vanish. This would violate the basic assumption of Proposition 6.5.

We bypass this difficulty by defining

$$
a_{i}^{\prime}= \begin{cases}a_{i} & \text { if } a_{i} \neq 1 \\ 2 & \text { if } a_{i}=1\end{cases}
$$

and

$$
b_{i}^{\prime}= \begin{cases}b_{i} & \text { if } b_{i} \neq 1 \\ 2 & \text { if } b_{i}=1\end{cases}
$$

The sets described above, obtained by replacing $a_{i}$ and $b_{i}$ by $a_{i}^{\prime}$ and $b_{i}^{\prime}$, remain equal. In order to see this, observe that if $a_{1}=1$, then the elements containing $a_{1}$ are

$$
\left\{\frac{a_{1}-1}{n}, \frac{a_{1}}{n}, \ldots, \frac{a_{1}+n-2}{n}\right\} ;
$$

these are replaced by

$$
\left\{\frac{a_{1}}{n}, \frac{a_{1}+1}{n}, \ldots, \frac{a_{1}+n-1}{n}\right\},
$$

which amounts to simply replacing $a_{1}$ by $a_{1}+1$. Theorem 6.6 shows that $a_{i}^{\prime}=b_{i}^{\prime}$. Whether $a_{i}$ and $b_{i}$ are both equal to 1 or both different from 1, we still conclude that $a_{i}=b_{i}$. This contradicts our original assumption. In the case where $a_{i}^{\prime}=b_{i}^{\prime}$ and $a_{i}=1$, we conclude that $b_{i}=2$. Similarly, if $a_{i}^{\prime}=b_{i}^{\prime}$ and $b_{i}=1$, then we obtain $a_{i}=2$. Therefore, any instance where $a_{i}=1$ produces the valid pair $\{1,2\}$, and any instance where $b_{i}=1$ leads to $\{2,1\}$. Using the fact that there are no common parameters in the numerator and denominator, we conclude that an eigenfunction must have the following structure.

TheOREM 6.16. Assume that

$$
f(x)=x \sum_{k=0}^{\infty} \frac{\left(a_{1}\right)_{k} \cdots\left(a_{p}\right)_{k}}{\left(b_{1}\right)_{k} \cdots\left(b_{q+1}\right)_{k}} x^{k}
$$

is an eigenfunction of $U_{n}$, with the usual normalization $b_{q+1}=1$. Then, either $a_{i}=2$ and $b_{i}=1$ whenever $1 \leq i \leq p$, or $a_{i}=1$ and $b_{i}=2$ whenever $1 \leq i \leq p$. 
In other words, if $f$ is an eigenfunction of any particular Hecke operator $U_{n}$, then

$$
f(x)={ }_{a} F_{a-1}\left(\mathbf{1}_{a}, \mathbf{2}_{a-1} ; x\right)=\sum_{k=0}^{\infty} \frac{1}{k^{a}} x^{k},
$$

or

$$
f(x)={ }_{a} F_{a-1}\left(\mathbf{2}_{a}, \mathbf{1}_{a-1} ; x\right)=\sum_{k=0}^{\infty} k^{a} x^{k},
$$

where $a \in \mathbb{N}$ and $\mathbf{1}_{a}$ stands for the $a$-vector $(1,1, \ldots, 1)$ and $2 b$ is defined analogously.

\section{The simultaneous eigenfunctions of $U_{n}$ for all $n$}

In this section we completely characterize those hypergeometric functions that are simultaneous eigenfunctions of $U_{n}$ for all $n$, and give an application to the theory of completely multiplicative functions.

What do the hypergeometric functions $f(x)=\sum_{k=1}^{\infty} c_{k} x^{k}$ that are simultaneous eigenfunctions of all of the linear operators $U_{n}$ look like? It turns out that there is a simple answer: they are precisely the polylogarithms and the rational functions $(x d / d x)^{a}(1 /(1-x))$, as given by the following theorem.

THEOREM 7.1. Let

$$
f(x)=\sum_{k=1}^{\infty} c_{k} x^{k}
$$

be a hypergeometric function with no constant term. Then $f$ is a simultaneous eigenfunction for the set of all Hecke operators $\left\{U_{n}\right\}_{n=1}^{\infty}$ if and only if

$$
f(x)=C \sum_{k=1}^{\infty} k^{a} x^{k},
$$

where $a \in \mathbb{Z}$ and $C \in \mathbb{C}$. In other words, either $f$ is a polylogarithm, or $f(x)=(x d / d x)^{a}(1 /(1-x))$.

Before proving this theorem, we prove an interesting corollary regarding a number-theoretic fact concerning hypergeometric coefficients that are completely multiplicative functions of the summation index.

COROLLARY 7.2. The hypergeometric coefficient

$$
c(n)=\frac{\left(a_{1}\right)_{n-1} \cdots\left(a_{p}\right)_{n-1}}{\left(b_{1}\right)_{n-1} \cdots\left(b_{q}\right)_{n-1}},
$$

is a completely multiplicative function of $n$ if and only if it is of the form $\mathrm{Cn}^{a}$ for some $a \in \mathbb{Z}$ and $C \in \mathbb{C}$.

PROOF. By the definition of completely multiplicative functions, we know that

$$
c(n k)=c(n) c(k)
$$


for all $k, n \in \mathbb{N}$. This implies that

$$
f(x)=\sum_{k=1}^{\infty} c(k) x^{k}
$$

is an eigenfunction of $U_{n}$, with eigenvalue $c(n)$. This holds for all $n$, thus it is a simultaneous eigenfunction. The result now follows from Theorem 7.1.

We recall that by definition $f$ is a simultaneous eigenfunction of all of the Hecke operators if and only if, for all $n \in \mathbb{N}$,

$$
U_{n} f=\lambda_{n} f .
$$

Treating $f$ as a power series without even considering its hypergeometric properties, we see that

$$
\sum_{k=1}^{\infty} c_{n k} x^{k}=\lambda_{n} \sum_{k=1}^{\infty} c_{k} x^{k},
$$

so that

$$
c_{n k}=\lambda_{n} c_{k} \text {. }
$$

This is true for all $k \geq 1$, so we can set $k=1$, which shows that

$$
c_{n}=\lambda_{n} c_{1} \text {. }
$$

This, in turn, is true for all $n$, and proves the following result.

LEMMA 7.3. Let

$$
f(x)=\sum_{k=1}^{\infty} c_{k} x^{k}
$$

be a power series with no constant term that is a simultaneous eigenfunction for the set of operators $\left\{U_{n}\right\}_{n=1}^{\infty}$ with respective eigenvalues $\left\{\lambda_{n}\right\}_{n=1}^{\infty}$. If $i \geq 2$, then

$$
c_{i}=\lambda_{i} c_{1}
$$

and thus

$$
f(x)=c_{1} \sum_{k=1}^{\infty} \lambda_{k} x^{k} .
$$

Proof of Theorem 7.1. Let us suppose now that $f$, as defined in (7.1), is a hypergeometric function. By Theorem 6.13,

$$
\lambda_{n}=n^{\gamma_{b}-\gamma_{a}},
$$

where $\gamma_{b}$ and $\gamma_{a}$ are defined in (6.5). This proves Theorem 7.1.

In our formal hypergeometric notation, we see that any simultaneous eigenfunction must be the polylogarithm

or the rational function

$$
\sum_{k=0}^{\infty} \frac{1}{k^{a}} x^{k}
$$

$$
\sum_{k=0}^{\infty} k^{a} x^{k}
$$


where $a$ is a nonnegative integer. In conclusion, we see that if a hypergeometric function is an eigenfunction for a single operator $U_{j}$, then it is automatically a simultaneous eigenfunction for all of the Hecke operators $U_{n}$, as $n$ varies over all positive integers. This situation stands in sharp contrast to the space of rational functions studied recently in [4-6].

\section{Acknowledgements}

The authors wish to thank the referees for a thorough report on an earlier version of the paper, and to thank Tai Ha for the proof of Theorem 6.16.

\section{References}

[1] G. E. Andrews, R. Askey and R. Roy, Special Functions, Encyclopedia of Mathematics and its Applications, 71 (Cambridge University Press, Cambridge, 1999).

[2] T. Apostol, Modular Functions and Dirichlet Series in Number Theory, 2nd edn, Graduate Texts in Mathematics, 41 (Springer, New York, 1990).

[3] C. D. Bennett and E. Mosteig, 'On the collection of integers that index the fixed points of maps on the space of rational functions', in: Tapas in Experimental Mathematics, Contemporary Mathematics, 457 (American Mathematical Society, Providence, RI, 2008), pp. 53-67.

[4] G. Boros, J. Little, V. Moll, E. Mosteig and R. Stanley, 'A map on the space of rational functions', Rocky Mountain J. Math. 35(6) (2005), 1861-1880.

[5] J. Gil and S. Robins, 'Hecke operators on rational functions. I', Forum Math. 17(4) (2005), 519-554.

[6] E. Mosteig, 'Fixed points of maps on the space of rational functions', Online J. Anal. Comb. 1 (2006), Article 2, 9 p.

[7] R. P. Stanley, Enumerative Combinatorics, Vol. 1, Cambridge Studies in Advanced Mathematics, 49 (Cambridge University Press, Cambridge, 1997).

VICTOR H. MOLL, Department of Mathematics, Tulane University, New Orleans, LA 70118, USA

e-mail: vhm@math.tulane.edu

SINAI ROBINS, Division of Mathematical Sciences, Nanyang Technological University, SPMS-MAS-03-01, 21 Nanyang Link, Singapore 637371, Singapore

e-mail: rsinai@ntu.edu.sg

KIRK SOODHALTER, Department of Mathematics, Temple University, Philadelphia, PA 19122, USA

e-mail:ksoodha@temple.edu 